\title{
Robot Drives Diagnostics by Identifiability Criterion Based on State Matrix
}

\author{
S. A. Trefilov, Yu. R. Nikitin \\ Department of Mechatronic Systems, Kalashnikov Izhevsk State Technical University, Izhevsk, Russia
}

E-mail: nikitin@istu.ru

Received: 15.07.2019

\begin{abstract}
The paper discusses robot drives diagnostics and optimal decision-making algorithm according to the identifiability criterion based on discrete digital control model. We consider discrete control algorithm for quality criterion that minimizes the energy of control and displacement. The optimal control algorithm is based on the Riccati equation solving for control system with modified state and control matrices. The criteria of observability, controllability and identifiability of robot drives are considered as rank function of the extended matrix with measurement matrix. An algorithm is proposed for calculating the criterion for identifiability of nonlinear control system in discrete linearization variant is proposed. Decision theory is applied for the robot drives diagnosis. It is suggested or we suggest to use identification in terms of mathematical model compliance to object operation results. A robot drives control using discrete vector-matrix algorithm involves calculating the state matrix at each step. Consequently, the expanded matrix determinant is calculated at each step and is compared with some constant that numerically divides the space of state matrices. Therefore, robot drives operation allows its identification. As the identification algorithm optimality criterion was chosen the optimal decision making criterion in combination with the identifiability criterion for the optimal control algorithm by the quadratic form criterion minimum. The vector-matrix model of robot drives in the state space is presented, taking into relative account state measuring accuracy of the informationmeasuring subsystem of robot drives. The drive model was developed in the Russian software package "Dynamic Simulation of Technical Systems SimInTech". It proposed to determine the identifiability criterion for practical tasks. The criterion of optimal decision making (threshold) can be chosen depending on the a priori data on the loss matrix and the probabilities of the hypothesis about the object mathematical model correspondence to the results of operation and the alternative - not about the correspondence of the model and experiment. In this paper, the identifiability conditions are considered not only in relation to the rank of the extended matrix $[\mathrm{C}, \mathrm{AC}, \ldots]$, but also as a condition for ensuring the accuracy of the model with respect to the object. It is proposed to model the identifiability threshold by exhaustive search of the object states for this model.
\end{abstract}

Keywords: identification, diagnostics, robot drives, state space, discrete model, optimal algorithm, Riccati equation, Cauchy matrix, Bayes criterion

\section{INTRODUCTION}

A robot drives work in extreme conditions. An effective diagnostic system is required to provide for high reliability robot drives. An optimal algorithm is proposed for deciding on robot drives identifiability, based on discrete digital control model. The diagnostic process de-

(C) S. A. Trefilov, Yu. R. Nikitin, 2019 
termines the adequacy of the model applicability to the object. The algorithm is based on calculating the value of the identifiability criterion for discrete non-linear model of robot drives in the state space.

The identification method in the state space has been actively developing over the past two decades. P. Eykhoff is one of the first who performed the theoretical identification justification, as well as developed algorithms and methods of identification [1,2]. The works of the following authors are devoted to the dynamic systems identification research: D. Graupe [3], L. Ljung [4], E. P. Sage and J. L. Melsa [5, 6], and among Russian authors - Ya. Z. Tsypkin [7], N. S. Rybman [8], S. E. Steinberg [9] and others.

R. V. Beard developed a scheme for detecting defects based on observers [10]. H. L. Jones continued these studies and developed the Beard-Jones Fault Detection Filter [11]. In the 1980s and early 1990s, the main approaches of quantitative diagnostics were developed: an observer-based approach, a parameter estimation method, etc. Some important study in this direction are works of P. M. Frank [12], R. Isermann [13, 14]. Theoretically well-substantiated developed techniques are classic diagnostic methods. These techniques are based on analytical redundancy, which is a model describing the diagnosed technical system.

\section{SeTting THE RESEARCH TASK}

A robot drives are represented by nonlinear differential control and observation equations in the state space.

$$
\begin{aligned}
& (t)+\mathbf{B}(t) \mathbf{u}(t), \\
& \mathbf{y}_{k}=\mathbf{C}_{k} \mathbf{x}_{k},
\end{aligned}
$$

where $\mathbf{A}(t)$ is a functional matrix of size $n \times n$, called the matrix of the system (object) state; $\mathbf{B}(t)$ is a functional matrix of size $n \times r$, called the control (input) matrix; $\mathbf{C}(t)$ is a functional matrix of size $m \times n$, called the state exit matrix or measurement matrix.

In general, when at least one of the matrix $\mathbf{A}(t), \mathbf{B}(t), \mathbf{C}(t)$ is time dependent, the task is nonlinear and has only particular solutions.

To find the state equation, we represent equations (1) in a discrete form, the discretization time $\Delta t$ tends to zero, and the trajectory on each discrete segment is linear. We write equations (1) in the form

$$
\begin{aligned}
\frac{\mathbf{x}_{k+1}-\mathbf{x}_{k}}{\Delta t} & =\mathbf{A}_{k} \mathbf{x}_{k}+\mathbf{B}_{k} \mathbf{u}_{k}, \\
\mathbf{y}_{k} & =\mathbf{C}_{k} \mathbf{x}_{k},
\end{aligned}
$$

Multiply the left and right sides of the first equation (2) by $\Delta t$, we get

$$
\mathbf{x}_{k+1}=\tilde{\imath}
$$

where $\tilde{i} \quad+\mathbf{E}, \tilde{]}$

This equation relates the transition of the system from the state $\mathbf{x}_{k}$ to the state $\mathbf{x}_{k+1}$. On the segment $\Delta t$, we take the values of the matrices $\mathbf{A}_{k}, \mathbf{B}_{k}$, and $\mathbf{C}_{k}$ to be constant. Find the solution to equation (3). For convenience, we will remove the "wavy line" sign in subsequent entries. 
The quadratic quality functional determining the control and displacement energy is expressed as follows

$$
I=\frac{1}{2} \int_{t_{0}}^{t_{f}}\left(\mathbf{x}^{\mathrm{T}} \mathbf{Q} \mathbf{x}+\mathbf{u}^{\mathrm{T}} \mathbf{G u}\right) d t,
$$

where $\mathbf{Q} \geq 0$ and $\mathbf{G}>0$ are arbitrarily defined matrices.

The equation solution (1) for the quality criterion (4), which minimizes the energy of control and displacement. It is determined by the following expression $[15,16]$.

$$
\begin{gathered}
\mathbf{u}=-\mathbf{G}^{-1} \mathbf{B}^{\mathrm{T}} \mathbf{K} \mathbf{x}, \\
\mathbf{x}=-\mathbf{C}^{-1} \mathbf{y},
\end{gathered}
$$

where $\mathbf{K}$ is the Cauchy matrix, $\mathbf{K}=\mathbf{K}^{\mathrm{T}}$, which can be found by solving the Riccati equation [15].

$$
-\mathbf{l} \quad{ }^{\mathrm{T}} \mathbf{K}+\mathbf{K}^{\mathrm{T}} \mathbf{A}-\mathbf{K}^{\mathrm{T}} \mathbf{B} \mathbf{G}^{-1} \mathbf{B}^{\mathrm{T}} \mathbf{K}
$$

where $\mathbf{K}\left(t_{f}\right)=0$.

Cauchy Matrix:

$$
\mathbf{K}=\left[\begin{array}{ccccc}
k_{11} & k_{12} & \ldots & k_{1(n-1)} & k_{1 n} \\
k_{21} & k_{22} & \ldots & k_{2(n-1)} & k_{2 n} \\
\ldots & \ldots & \ldots & \ldots & \ldots \\
k_{(n-1) 1} & k_{(n-1) 2} & \ldots & k_{(n-1)(n-1)} & k_{(n-1) n} \\
k_{n 1} & k_{n 2} & \ldots & k_{n(n-1)} & k_{n n}
\end{array}\right]
$$

where $k_{i j}=k_{j i}$, should be positively defined, since as positive definite matrices $\mathbf{Q}$ and $\mathbf{G}$ is used in the quadratic quality functional (4), which is also positive.

Matrices $\mathbf{Q}$ and $\mathbf{G}$ are chosen arbitrary. Matrices $\mathbf{Q}$ and $\mathbf{G}$ are selected by the assortment method, since they are not always possible to obtain a satisfactory solution of equation (5), when calculating the control vector $\mathbf{u}$. In $[15,16]$, it is proposed to select these matrices by assortment or simulation.

Thus, the driver control is carried out by solving (6) according to the model (5) by calculating at each step the state matrix $\mathbf{A}_{k}$ and the control matrix $\mathbf{B}_{k}$ (3).

We assume that the matrix $\mathbf{C}^{-1}=\mathbf{C}_{k}^{-1}$ at each step $k$ does not change, is determined by the information-measuring system, can be represented as

$$
\mathbf{C}^{-1}=\left[\begin{array}{cccc}
1+\xi_{1} & 0 & \ldots & 0 \\
0 & 1+\xi_{2} & \ldots & 0 \\
\ldots & \ldots & \ldots & \ldots \\
0 & 0 & \ldots & 1+\xi_{n}
\end{array}\right],
$$

where $\xi_{n}=\left[\begin{array}{llll}\xi_{1} & \xi_{2} & \ldots & \xi_{n}\end{array}\right]^{\mathrm{T}}$ is random vector, that reflects the random nature of measurements by the information-measuring system, that is part of the drives [17, 18]. 


\section{DRIVE IDENTIFICATION}

Consider the issue of identifying drives in terms of analyzing expression (9), where at each step of linearization the criterion of identifiability and observability is the rank of the extended matrix.

$$
\operatorname{rank}\left[\begin{array}{lllll}
\mathbf{C}_{k}^{\mathrm{T}} & \mathbf{A}_{k}^{\mathrm{T}} \mathbf{C}_{k}^{\mathrm{T}} & \left(\mathbf{A}_{k}^{\mathrm{T}}\right)^{2} \mathbf{C}_{k}^{\mathrm{T}} & \cdots & \left(\mathbf{A}_{k}^{\mathrm{T}}\right)^{n-1} \mathbf{C}_{k}^{\mathrm{T}}
\end{array}\right]=n .
$$

The matrix $\mathbf{C}_{k}^{\mathrm{T}}$ is completely determined by the information-measuring system, that is, by relative measurement error or accuracy class. We write the model of informationmeasuring system in the form

$$
\mathbf{y}_{k}=\mathbf{C}_{k} \mathbf{x}_{k},
$$

or

$$
\left[\begin{array}{l}
y_{1} \\
y_{2} \\
\ldots \\
y_{n}
\end{array}\right]=\left[\begin{array}{cccc}
1+h_{1} \xi_{1} & 0 & \ldots & 0 \\
0 & 1+h_{2} \xi_{2} & \ldots & 0 \\
\ldots & \ldots & \ldots & \ldots \\
0 & 0 & \ldots & 1+h_{n} \xi_{n}
\end{array}\right]\left[\begin{array}{l}
x_{1} \\
x_{2} \\
\ldots \\
x_{n}
\end{array}\right],
$$

where $h_{i}$ is the relative measurement error, $\xi_{i}$ is implementation of a normally distributed random variable with standard deviation $\sigma_{i}=\frac{h_{i}}{3}, i=\overline{1, n}$.

Then for the maximum downward errors in the worst case for all measuring channels, given the continuous and infinite nature of the implementation of a normally distributed random variable, assuming that most of the values fall within the interval $-3 \sigma_{i} \leq \xi_{i} \leq 3 \sigma_{i}$, $i=\overline{1, n}$, we can write approximately

$$
\mathbf{C}_{n}=\left[\begin{array}{cccc}
1-h_{1} & 0 & \ldots & 0 \\
0 & 1-h_{2} & \ldots & 0 \\
\ldots & \ldots & \ldots & \ldots \\
0 & 0 & \ldots & 1-h_{n}
\end{array}\right]
$$

In our case, the measurement channels are independent and the determinant of the matrix will be equal to

$$
\operatorname{det} \mathbf{C}_{n}^{T}=\left(1-h_{1}\right)\left(1-h_{2}\right) \ldots\left(1-h_{n}\right) .
$$

Open the brackets in (12), exclude the terms of the second and higher order of smallness, we get

$$
\operatorname{det} \mathbf{C}_{n}^{\mathrm{T}}=1-h_{1}-h_{2}-\ldots-h_{n}=1-\sum_{i=1}^{n} h_{i}
$$

If the relative accuracy of all measuring channels is the same $h_{i}=h, i=\overline{1, n}$, then from (13), we get

$$
\operatorname{det} \mathbf{C}_{n}^{\mathrm{T}}=1-n h .
$$

Similarly, for maximum errors in a big way in the worst case for all measuring channels we can write 


$$
\begin{gathered}
\operatorname{det} \mathbf{C}_{n}^{\mathrm{T}}=1+h_{1}+h_{2}+\ldots+h_{n}=1+\sum_{i=1}^{n} h_{i} . \\
\operatorname{det} \mathbf{C}_{n}^{\mathrm{T}}=1+n h .
\end{gathered}
$$

The value of the determinant in (14) and (15) is always greater than one, since the value of relative accuracy is always positive,

$$
h_{i}>0, i=\overline{1, n} .
$$

Thus, the identifiability condition (10) in the case

$$
\operatorname{det} \mathbf{A}_{k}^{\mathrm{T}}(n)^{i}>0, i=\overline{1, n}
$$

will be the condition

$$
\operatorname{det} \mathbf{C}_{n}^{\mathrm{T}}=1-\sum_{i=1}^{n} h_{i}>0
$$

or

$$
\sum_{i=1}^{n} h_{i}<1
$$

The following is DC motor model in the state space, depending on the accuracy of measuring the state matrix under the assumption that the given path accuracy is no worse than $10 \%$. Going beyond this value will be considered a loss of identifiability, that is, the model does not match the object.

Figure 1 shows the scheme for calculating the model output parameters at different accuracy of measuring the state vector of the DC motor in a vector-matrix form with the "State variables" block in the dynamic modeling environment of technical systems SimInTech.

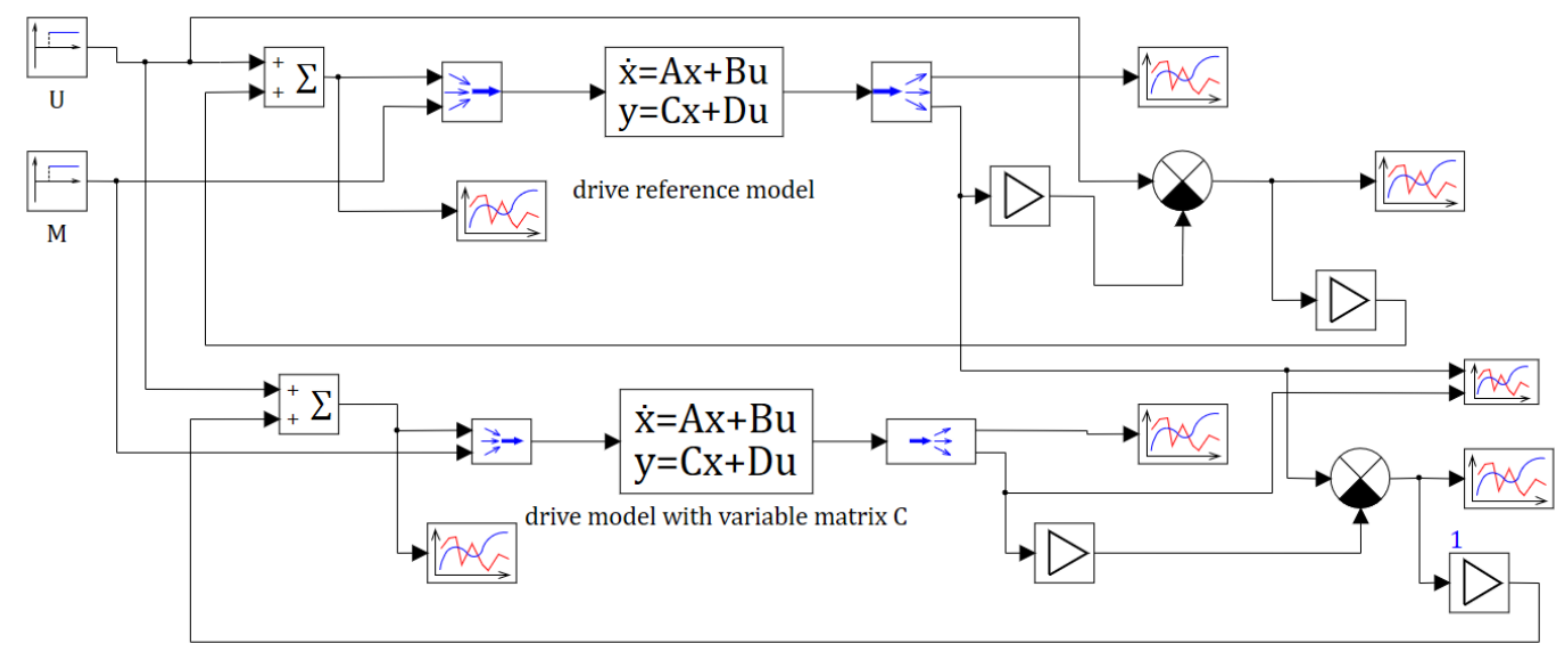

Figure 1. DC motor model with a reference and variable measuring matrix

The simulation results at torque $M=1.91 \mathrm{~N}$, a relative measurement error $h_{1}=0$ (reference measuring matrix without measurement errors), $h_{2}=0.1$ (measuring matrix in the presence of a relative measurement error of 0.1 ) are presented in Fig. 2. 


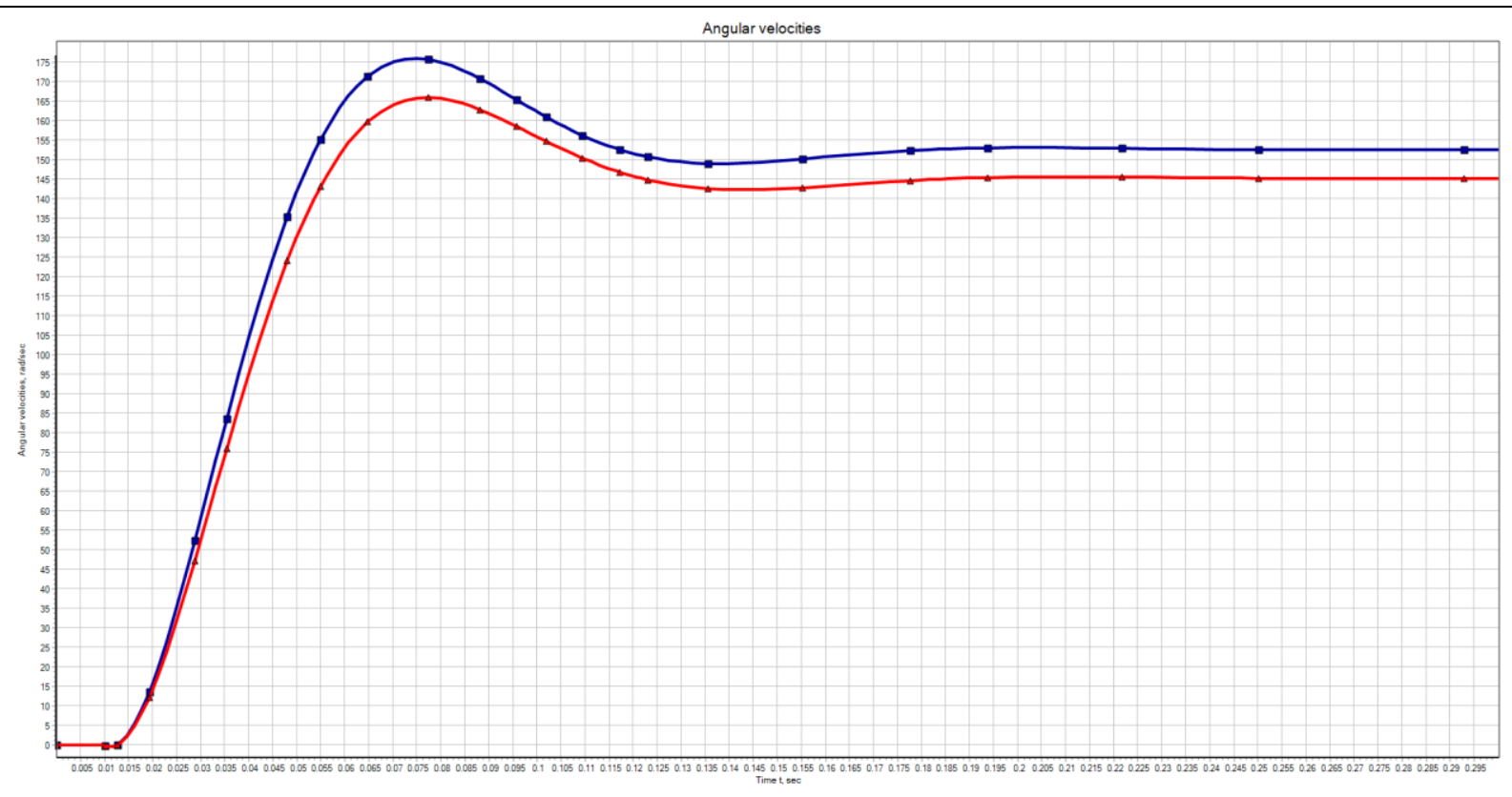

Figure 2. Simulation results at torque $M=1.91 \mathrm{~N}, h_{1}=0$ (reference measuring matrix), $h_{2}=0.1$ (variable measuring matrix)

Fig. 3 shows the dependence of the relative difference in the angular velocities of the reference model and the model with a modified measurement matrix.

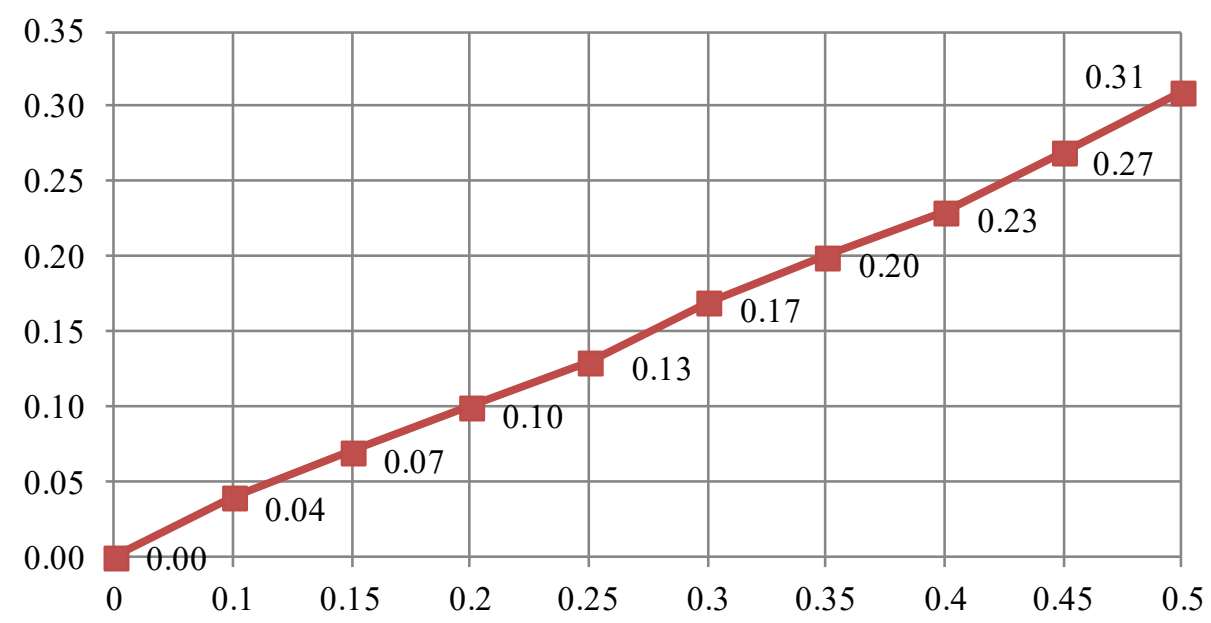

Figure 3. Dependence of the relative difference in the angular velocities of the reference model and the model with modified observation matrix (the abscissa axis is $h$, the ordinate axis is the relative difference of angular velocities)

Considering that in many practical problems of managing tasks, the dimensions of problems do not exceed ten, and the relative accuracy of measurement is equal to units of percent, it can be concluded that only the state matrices affect the identifiability

$$
\mathbf{A}_{k}^{\mathrm{T}}, k=\overline{1, n},
$$

which will ultimately determine the rank of the matrix (9). 
It is proposed for practical tasks to determine identifiability in the form

$$
\min \left(\operatorname{det} \mathbf{A}_{k}^{\mathrm{T}}(n)^{i} \operatorname{det} \mathbf{C}_{k}^{T}(n)\right)>\gamma .
$$

where $k$ is the step number in the nonlinear model; $n$ is the dimension of the model; $\gamma$ is the identifiability criterion chosen by modeling the state matrix for the cases when the parameters of matrix $\mathbf{A}$ are exited from the space of realizable values of a serviceable system.

\section{DRIVE DiAgNOSTICS}

Apply the decision theory $[19,20]$ to the choice of the threshold in (17). Let's write the source data for this task:

- $S_{0}$ is system state corresponding to identifiability;

- $S_{1}$ is system state corresponding to non-identifiability;

- $X_{0}$ is the space of samples corresponding to condition (17) of the identifiability of the system with probability $P\left\{x \in X_{0} \mid S_{0}\right\}$;

- $X_{1}$ is the sample space corresponding to condition (17) of the system's nonidentifiability with probability $P\left\{x \in X_{1} \mid S_{1}\right\}$;

- $q$ is the probability that the state of the system corresponds to the state $S_{0}$;

- $p$ is an alternative, i.e. $p=1-q$ is the probability that the state of the system corresponds to the state $S_{1}$;

- $H_{0}$ is hypothesis about the belonging of the observed vector $\mathbf{x}=\left(x_{1}, x_{2}, \ldots, x_{n}\right)^{\mathrm{T}}$ to the state $S_{0}$;

- $H_{1}$ is hypothesis about the belonging of the observed vector $\mathbf{x}=\left(x_{1}, x_{2}, \ldots, x_{n}\right)^{\mathrm{T}}$ to the state $S_{1}$;

- $\gamma_{0}$ is the decision to accept the true hypothesis $H_{0}$ with probability $P\left\{\mathbf{x} \in X_{0} \mid S_{0}\right\}$;

- $\gamma_{1}$ is the decision to accept the true hypothesis $H_{1}$ with probability $P\left\{\mathbf{x} \in X_{1} \mid S_{1}\right\}$;

- $W_{n}\left(\mathbf{x} \mid S_{0}\right)$ is conditional density function for samples corresponding to the state $S_{0}$;

- $W_{n}\left(\mathbf{x} \mid S_{1}\right)$ is conditional density function for samples corresponding to the state $S_{1}$;

$-l(x)=\frac{W_{n}\left(\mathbf{x} \mid S_{0}\right)}{W_{n}\left(\mathbf{x} \mid S_{1}\right)}$ is likelihood statistics.

Then if the likelihood statistics

$$
l(x)=\frac{W_{n}\left(\mathbf{x} \mid S_{0}\right)}{W_{n}\left(\mathbf{x} \mid S_{1}\right)}>c,
$$

then we decide $\gamma_{0}$ on the correctness of the hypothesis $H_{0}$, that the observed vector (sample) $\mathbf{x}=\left(x_{1}, x_{2}, \ldots, x_{n}\right)^{\mathrm{T}}$ belongs to the state $S_{0}$, i.e. the robot drive is identifiable. Otherwise, the likelihood statistics

$$
l(x)=\frac{W_{n}\left(\mathbf{x} \mid S_{0}\right)}{W_{n}\left(\mathbf{x} \mid S_{1}\right)} \leq c,
$$


then we decide $\gamma_{1}$ on the correctness of the hypothesis $H_{1}$, that the observed vector (sample) $\mathbf{x}=\left(x_{1}, x_{2}, \ldots, x_{n}\right)^{\mathrm{T}}$ belongs to the state $S_{1}-$ the decision on the system's non-identifiability. Optimal decision-making on the selected criterion implies errors: $\alpha=P\left\{x \in X_{1} \mid S_{0}\right\}$ - decision making $\gamma_{1}$, although the state was true $S_{0}$ (error of the first kind) and $\beta=P\left\{x \in X_{0} \mid S_{1}\right\}$ decision-making $\gamma_{0}$, although the state was true $S_{0}$ (error of the second kind).

The threshold $c$ is selected as one of the decision methods. For example, for the Bayes method $[19,20]$, we can record the statistics of the correct decision on the identifiability of drives in the form

$$
l(x)=\frac{W_{n}\left(\mathbf{x} \mid S_{0}\right)}{W_{n}\left(\mathbf{x} \mid S_{1}\right)}>\frac{\prod_{01}-\prod_{00}}{\prod_{10}-\prod_{11}} \frac{q}{p},
$$

where $\prod_{00}, \prod_{11}, \prod_{01}, \prod_{10}$ - elements of the loss matrix, and $\prod_{00}, \prod_{11}$ are weights for correct decisions, and $\prod_{01}, \prod_{10}$ - for erroneous ones. For a simple loss matrix of identical probabilities $p$ and $q[19,20]$, we obtain the statistics of the correct decision on the identifiability of the system in a simpler form

$$
l(x)=\frac{W_{n}\left(\mathbf{x} \mid S_{0}\right)}{W_{n}\left(\mathbf{x} \mid S_{1}\right)}>1,
$$

which meets the maximum likelihood criterion.

Thus, solving together $(17,18,19)$ for a given threshold $c$, going through all possible values $\mathbf{x}=\left(x_{1}, x_{2}, \ldots, x_{n}\right)^{\mathrm{T}}$ from the space $X_{0}+X_{1}$ for all states $S_{0}$ and $S_{1}$, we obtain the value $\gamma$. Note that solution (17) presupposes the dependence of the matrix $\mathbf{A}_{k}^{\mathrm{T}}, k=\overline{1, n}$ on the state $\mathbf{x}=\left(x_{1}, x_{2}, \ldots, x_{n}\right)^{\mathrm{T}}$ and, therefore, it has greater computational complexity of the numerical solution. Considering the universality of the likelihood statistics $(18,19)$, the criterion of optimal decision making (threshold $c$ ) can be chosen depending on a priori data on the loss matrix and the probabilities of the hypothesis and alternative $[19,20]$.

\section{CONCLUSION}

Currently the development of control algorithms based on discrete models is a very important task, because almost all tasks of controlling practice of robots drives cannot be accurately represented by linear models and the solution of nonlinear models is generally absent. Discrete models of nonlinear systems of the form (3) presuppose variable matrices of state, control, and measurement. Matrices are defining an infinite number of the model variants. Therefore, some tools are needed to calculate of adequacy degree of mathematical models and real objects. This paper presents the optimal algorithm for identifying nonlinear complex systems based on a discrete digital control model. As the criterion for the optimality of the identification algorithm was chosen the criterion of optimal decision making in combination with the identifiability criterion for the optimal control algorithm by the minimum criterion of the quadratic form.

The paper investigates the influence of the parameters of the measurement matrix on the identifiability of the robot drive model based on the DC motor for control and diagnostic tasks. 
It is shown that at a threshold value $\mathrm{h}>0.2$ of the measurement matrix, the difference in the angular velocities of the reference model and the model with the modified observation matrix is more than $10 \%$, the drive model becomes unidentifiable, which leads to a loss of controllability and the inability to diagnose. To study the influence of the measurement matrix on the identifiability of the model, a vector-matrix model of the drive in the state space is developed taking into account viscous friction.

Identification criteria (16) and (17) allow to determine the models conformity degree to the control object by the model of the measuring matrix or by the models combination of the state matrix and the measuring matrix.

The threshold $\gamma$ in (17) is optimal and is calculated numerically by solving the system of inequalities $(17,18,19)$. It is possible to require, though it is not necessary, in the criterion (16) for the measurement matrix, that the sum of the relative errors be substantially less than one. The criterion of optimal decision making (threshold c) can be chosen depending on the apriori data by the loss matrix and the probabilities of the hypothesis and the alternative.

In this paper are new:

1) the conditions of identifiability are considered not only with respect to the rank of the extended matrix $[\mathrm{C}, \mathrm{AC}, \ldots]$, but also as a condition for ensuring the accuracy of the model with respect to the object;

2) it is proposed to model the identifiability threshold by exhaustive search of the state of an object for this model.

\section{ACKNOWLEDGMENT}

The reported study was funded by RFBR, project number 18-08-00772 A.

\section{REFERENCES}

1. Eykhoff, P. (1974). System identification: Parameter and state estimation. Wiley-Interscience, New York. $555 \mathrm{p}$.

2. Eykhoff, P. (Ed.). (1981). Trends and progress in system identification. Oxford, England: Pergamon. 402 p.

3. Graupe, D. (1976). Identification of system. New York: R. E. Krieger Publishing Company. 302 p.

4. Ljung, L. (1999). System identification. Theory for the user. 2nd ed. PTR Prentice Hall, Upper Saddle River. 609 p.

5. Sage, A., \& Melsa, J. (1971). System identification. New York: Academic press. 221 p.

6. Sage, A., \& Melsa, J. (1971). Estimation theory with applications to communications and control. New York: McGraw-Hill. 496 p.

7. Cypkin, Ja. Z. (1984). Osnovy informacionnoj teorii identifikacii [Fundamentals of identification information theory]. Moscow, USSR: Nauka. 320 p. (in Russian).

8. Rajbman, N. S. (1970). Chto takoe identifikacija? [What is identification?]. Moscow, USSR: Nauka. 118 p. (in Russian).

9. Shtejnberg, Sh. E. (1987). Identifikacija v sistemah upravlenija [Identification in control systems]. Moscow, USSR: Energoatomizdat. 80 p. (in Russian).

10. Beard, R. V. (1971). Failure accommodation in linear system through selfreorganization (PhD thesis). Massachusetts, USA: MIT. 376 p.

11. Jones, H. L. (1973). Failure detection in linear systems (PhD thesis). Massachusetts, USA: MIT. 459 p.

12. Frank, P. M. (1990). Fault diagnosis in dynamic systems using analytical and knowledge-based redundancy: A survey and some new results. Automatica, 26, 459-474. doi: 10.1016/0005-1098(90)90018-D 
13. Isermann, R. (2006). Fault-diagnosis systems: An introduction from fault detection to fault tolerance. Springer-Verlag Berlin Heidelberg. 475 p. doi: 10.1007/3-540-30368-5

14. Isermann, R. (2017). Combustion engine diagnosis. Model-based condition monitoring of gasoline and diesel engines and their components. Springer Vieweg. 303 p. doi: 10.1007/978-3-662-49467-7

15. Sage, A.P., \& White, C.C. III. (1993). Optimum systems control. Englewood Cliffs, USA: Prentice Hall. $392 \mathrm{p}$.

16. Krasovskij, A. A. (1987) Spravochnik po teorii avtomaticheskogo upravlenija [Handbook of automatic control theory]. Moscow, USSR: Nauka, Gl. red. fiz.-mat. lit. 712 p. (in Russian).

17. Trefilov, S., \& Nikitin, Y. (2018). Automatic warehouses with transport robots of increased reliability. Acta Logistica, 5(1), 19-23. doi: 10.22306/al.v5i1.86

18. Trefilov, S. A., Kalinkin, A. A., \& Nikitin, Y. R. (2007). High-maneuverable transport robot. University Review, 1(2), 34-39. Trencin, Slovakia: Alexander Dubcek University of Trencin; Izhevsk, Russia: Izhevsk State Technical University.

19. Levin, B. R. (1968). Teoreticheskie osnovy statisticheskoj radiotehniki [Theoretical foundations of statistical radio engineering]. Vol. 2. Moscow, USSR: Sovetskoe radio. 504 p. (in Russian).

20. Pugachev, V. S., \& Sinicyn, I. N. (1990). Stohasticheskie differencial'nye sistemy. Analiz i fil'tracija [Stochastic differential systems. Analysis and filtering]. Moscow, USSR: Nauka. 632 p. (in Russian). 